\title{
Resveratrol activates DNA damage response through inhibition of polo-like kinase 1 (PLK1) in natural killer/T cell lymphoma
}

\author{
Xianxian Sui ${ }^{1 \#}$, Canjing Zhang ${ }^{2 \#}$, Yudong Jiang ${ }^{3}$, Jianan Zhou ${ }^{4}$, Chen $\mathrm{Xu}^{5}$, Feng Tang ${ }^{6}$, Bobin Chen ${ }^{7}$, \\ Huiwen $\mathrm{Xu}^{7}$, Songmei Wang ${ }^{1}$, Xuanyi Wang ${ }^{2}$ \\ ${ }^{1}$ Laboratory of Medical Molecular Biology, Experimental Teaching Center, School of Basic Medical Sciences, Fudan University, Shanghai, China; \\ ${ }^{2}$ Key Laboratory of Medical Molecular Virology of Ministry of Education \& Ministry of Health, and Institutes of Biomedical Sciences, Fudan \\ University, Shanghai 200032, China; ${ }^{3}$ Shanghai Medical College, Fudan University, Shanghai, China; ${ }^{4}$ The Fifth People's Hospital of Shanghai, \\ Fudan University, Shanghai, China; ${ }^{5}$ Department of Pathology, Zhongshan Hospital, Fudan University, Shanghai, China; ${ }^{6}$ Department of Pathology, \\ Huashan Hospital, Fudan University, Shanghai, China; ${ }^{7}$ Department of Hematology, Huashan Hospital, Fudan University, Shanghai, China \\ Contributions: (I) Conception and design: B Chen, S Wang, X Wang; (II) Administrative support: X Sui; (III) Provision of study materials or patients: \\ C Xu, F Tang; (IV) Collection and assembly of data: X Sui, C Zhang, J Zhou; (V) Data analysis and interpretation: X Sui, C Zhang, C Xu, F Tang; (VI) \\ Manuscript writing: All authors; (VII) Final approval of manuscript: All authors. \\ \#These authors contributed equally to this work. \\ Correspondence to: Songmei Wang, MD, PhD. Laboratory of Medical Molecular Biology, Experimental Teaching Center, School of Basic Medical \\ Sciences, Fudan University, 131 Dong'an Rd., Xuhui District, Shanghai, China. Email: smwang2@fudan.edu.cn; Xuanyi Wang, MD, PhD. Key \\ Laboratory of Medical Molecular Virology of Ministry of Education \& Ministry of Health, and Institutes of Biomedical Sciences, Fudan University, \\ 131 Dong’an Rd., Xuhui District, Shanghai 200032, China. Email: xywang@shmu.edu.cn.
}

Background: Extranodal natural killer/T cell lymphoma (NKTCL) is a highly aggressive non-Hodgkin lymphoma with a poor prognosis. Resveratrol (REV), a natural nontoxic pleiotropic agent, has antitumor effects, yet not being studied in NKTCL.

Methods: We performed immunohistochemical (IHC) staining with NKTCL tumor tissues. Apoptosis and cell cycle of NKTCL cell line NK-92 were detected by using flow cytometry. Then we detected the cellular expression level of polo-like kinase 1 (PLK1) and key molecules in DNA damage response (DDR) pathway by using RNA sequencing (RNA-seq) technology, real-time PCR, and Western blot.

Results: In this study, we found distinguishingly expressed phosphorylated ataxia telangiectasia mutated (ATM) in human NKTCL tumor tissues compared to normal lymph nodes samples. But low levels of phosphorylated checkpoint kinase $2(\mathrm{Chk} 2)$ and phosphorylated $\mathrm{p} 53$ were shown, suggesting that DDR pathway is blocked midway in NKTCL. REV inhibited the proliferation of NK-92 cells in a time- and dose-dependent manner, arrested cell cycle at G1 phase, and induced mitochondrial apoptosis. PLK1 was inhibited in both mRNA and protein levels by REV in NK-92 cells. At the same time, phosphorylation levels of Chk2 and p53 were upregulated.

Conclusions: DDR pathway plays an important role in the pathogenesis of NKTCL. REV shows antiNKTCL activity. The inhibition of PLK1 and the activation of DDR are vital for REV induced tumor cell apoptosis.

Keywords: Extranodal natural killer/T cell lymphoma (NKTCL); resveratrol (REV); DNA damage response pathway (DDR pathway); polo-like kinase 1 (PLK1); checkpoint kinase 2 (Chk2)

Submitted Dec 12, 2019. Accepted for publication Apr 27, 2020.

doi: $10.21037 /$ atm-19-4324

View this article at: http://dx.doi.org/10.21037/atm-19-4324 


\section{Introduction}

Extranodal natural killer/T cell lymphoma (NKTCL) is a rare subtype of non-Hodgkin lymphoma (NHL), and is highly associated with Epstein-Barr virus (EBV) infection (1). It is more common in Asia and South America (up to 10\% of NHL) than in North American and European countries (up to 1\%) (2). Most cases of NKTCL (80-90\%) are first found as extranodal involvement, mainly in the nasal cavity and adjacent sites (3). Radiotherapy used to be the first choice for localized NKTCL, while there are also studies showing that concurrent chemoradiotherapy (CCRT) with platinum or L-asparaginase and without anthracyclines has improved the prognosis of patients with NKTCL. Even so, nearly half of the patients still experience disease progression or relapse. The prognosis of patients with advanced or relapsed NKTCL remains unsatisfactory (4). Therefore, effective treatment exploration is necessary for NKTCL. The pathogenesis of NKTCL is yet unknown. However, dysregulation of pathways that concerning cell fate were clarified in NKTCL, including Akt, NF-kB, and JAK-STAT pathways. To our notice, the delay in the repair of DNA double strand break (DSB) and the defect of $\mathrm{p} 53$ accumulation in NKTCL cell lines after infrared radiation (IR) and ultraviolet (UV) exposure suggest the role of DNA damage response (DDR) in the initiation and progression of NKTCL (5).

DDR is a complex network to detect DNA lesions and to further initiate DNA repair, regulate cell cycle, or induce cell apoptosis. DDR pathway consists of "sensors", "transducers" and "effectors". The "sensors", for example, the Mre11-Rad50-Nbs1 (MRN) complex, detect DNA damage. The "transducers" are kinases including ataxia telangiectasia mutated (ATM) and ataxia telangiectasia and rad3-related (ATR) which recruit the following "effectors" to repair DNA, arrest cell cycle or induce apoptosis. Substrates of ATM and ATR, including Chk1, Chk2, p53 and H2A histone family member X (H2A.X), are effectors (6). DDR dominates cell fate considering the severity of DNA damage and the survival potentiality of cells (7). Mild DNA damage can be repaired by DDR, which demands a transient cell cycle arrest. In contrast, severe DNA damage can ultimately lead to cell death, avoiding potential threaten (8).

There are several regulatory factors closely related to DDR pathway, among which, Polo-like kinase 1 (PLK1) is a cell cycle regulator. When DNA damage occurs, PLK1 is inhibited by activated ATM, leading to cell cycle arrests at multiple points in mitosis (9). PLK1 is found to be overexpressed in a variety of human tumors and its overexpression closely related to tumorigenesis (10). The suppression of PLK1 can induce DDR and cell apoptosis in cancer cell lines $(11,12)$. Thus, PLK1 is a potential target for cancer therapy. Chemotherapy and radiotherapy kill tumor cells mostly by inducing DNA damage (13). However, the side effects including serum enzyme elevations, liver injury, gastrointestinal reactions could be intolerable and sometimes severe. One promising approach to assist chemotherapy could be the combination use of drugs targeting DDR with less toxicity so that the same effectiveness could be achieved with less chemotherapy drugs dosage. The traditional Chinese drug, resveratrol (REV), may be competent for this job.

REV, a polyphenolic compound rich in grapes, blueberries, and peanuts, has wide pharmacological activities including antioxidant, anti-inflammatory, cardio-protection, neuro-protection, and especially, antitumor activities (14). REV interferes with the initiation, promotion and progression stages of carcinogenesis, sensitizes tumor cells to standard chemotherapeutic agents and reverses multidrug resistance $(15,16)$. Studies focusing on its antitumor mechanisms showed that REV can induce DDR to kill tumor cells (17). Due to its low toxicity in animal models and in humans, REV can be a potent adjuvant to sensitize tumor cells to antitumor therapies and to ameliorate the side effects (18-20).

In present study, we found that REV inhibits NKTCL cell line NK-92 cells growth and induces cell cycle arrest and apoptosis by reactivating DDR pathway, which is blocked in NKTCL. Inhibition of PLK1 may be the key mechanism of REV to activate DDR.

\section{Methods}

\section{Cells}

NK-92 cells, an EBV-positive NK cell line derived from peripheral blood mononuclear cells of a patient with malignant NHL, was purchased from ATCC (Manassas, USA). Cells were cultured in $\alpha$-MEM medium (Hyclone, Utah, USA) containing $12.5 \%$ horse serum (Gibco, Pittsburgh, USA) and $12.5 \%$ fetal bovine serum (Gibco, Pittsburgh, USA), supplemented with $100 \mathrm{U} / \mathrm{mL}$ recombinant human interleukin-2 (IL-2, Peprotech, NJ, USA). 
Table 1 The clinical and pathological characteristics of specimens

\begin{tabular}{|c|c|c|c|c|c|c|c|c|c|}
\hline Samples & Age (years) & Sex & Biopsy site & T clonality & CD56 & CD2 & $\mathrm{CD} 3 \varepsilon$ & TIA1 & Ki67 \\
\hline Case 2 & 59 & M & Nasal cavity & - & $-/+$ & $-/+$ & - & + & $70 \%+$ \\
\hline Case 3 & 73 & M & Front end of right inferior turbinate & - & $\mathrm{P}_{+}$ & + & + & $\mathrm{P}+$ & $70 \%+$ \\
\hline Case 4 & 43 & $\mathrm{~F}$ & Right of inferior turbinate & + & + & $\mathrm{P}+$ & - & - & $60 \%+$ \\
\hline Case 6 & 63 & M & Small intestine & + & + & + & + & - & $80 \%+$ \\
\hline Case 7 & 44 & M & Left testis & + & + & $-/+$ & $\mathrm{P}+$ & + & $70 \%+$ \\
\hline Case 8 & 63 & $\mathrm{M}$ & Left upper limb & - & $\mathrm{P}+$ & $\mathrm{P}+$ & $-/+$ & + & $70 \%+$ \\
\hline Case 9 & 58 & M & Nasopharynx & - & + & + & + & + & $70 \%+$ \\
\hline Case 12 & 37 & $M$ & Right hemicolon & + & + & + & + & - & $80 \%+$ \\
\hline
\end{tabular}

M, male; F, female; $P_{+}$, part of positive; -/+, a little of positive.

\section{Specimens}

Tissue slices (histological section) of NKTCL tumor tissues from twelve diagnosed NKTCL patients and six normal lymph nodes from non-lymphoma patients as healthy controls were collected from pathology department of Huashan Hospital from 2012 to 2016. This study was approved by medical ethics committee of school of Basic Medical Sciences, Fudan University. The clinical and pathological characteristics were summarized in Table 1.

\section{Data mining}

We employed $\mathrm{R}$ language (3.5.2) to mine data from the online tissue chip database Array Express. The annotation package for the E-TABM-702 tissue chip was hgu133plus2. $\mathrm{db}$, by which we translated probe ID into gene names.

\section{CCK-8 assay/cell proliferation}

NK-92 cells were treated with DMSO or REV (Sigma, louis, USA) at different concentrations for different time. Cell viability was measured by cell counting kit- 8 assay (DOJINDO, Japan) and cell viability ratio were calculated using equation below:

cell viability ratio $(\%)=[\mathrm{OD}(\mathrm{REV})-\mathrm{OD}($ Blank $)] /[\mathrm{OD}$ (control) - OD (Blank)] ×100\%.

\section{Flow cytometry assay}

Cell cycle distribution was measured by propidium iodide (PI, KeyGEN, Nanjing, China) staining, and cell apoptosis was measured by FITC Annexin V Apoptosis Detection Kit (BD, NJ, USA) according to manufacturer's introduction on FACSCalibur (BD, NJ, USA) using CELLQuest software (Becton Dickinson, Mountain View, CA).

\section{Gene expression profiles of REV treated NK-92 cells}

Gene expression profiles of NK-92 cells was measured using RNA-seq analysis. Cells were treated with DMSO or REV for $6 \mathrm{~h}$. Each group consisted with three replicates. The mRNA of NK-92 cells was enriched and purified. Double strand cDNA was transcribed from fragmented mRNA by N6 random primer and was phosphorylated at $5^{\prime}$ end while "A" stickiness and "T" adaptor were added at 3' end. The final cDNA library was created by PCR and sequenced on BGISEQ-500 Instrument (BGI, Shenzhen, China). Quality control of raw data was performed by data filtering and reads mapping.

\section{SDS-PAGE and Western blot analysis}

The proteins retrieved from cells lysed with RIPA lysis buffer (Beyotime, Shanghai, China) supplemented with 
Phos-stop (Roche, Basel, Switzerland) and $10 \mathrm{mM}$ PMSF (Beyotime, Shanghai, China). The protein concentrations were measured by BCA protein assay kits (Beyotime, Shanghai, China). Proteins prepared in equivalent concentration were separated by SDS-PAGE and transferred to PVDF membranes (Millipore, Bedford, MA). The membranes were blocked with $5 \%$ non-fat milk at room temperature for $1 \mathrm{~h}$ and then incubated with primary antibodies specific to target proteins overnight at $4{ }^{\circ} \mathrm{C}$. After being washed in TBST, membranes were incubated with HRP-labelled goat anti-rabbit or mouse secondary antibody (1:4,000, CWBIO, Shanghai, China). Chemiluminescence image analysis system (Tannon, Shanghai, China) was used to visualize the protein bands. $\beta$-actin or GAPDH was employed as the internal reference.

The primary antibodies of phospho-Akt (pAkt) (Ser473), Akt, phospho-Stat3 (pStat3) (Tyr705), Stat3, phospho-Chk2 (pChk2) (Thr67), phospho-p53 (pp53) (Ser15), phosphoPLK1 (pPLK1) (Thr210) and PLK1 were purchased from Cell Signaling Technology (Boston, USA). Anti-p53 was purchased from EMD Millipore (Temecula, USA). AntiZEBRA was purchased from Santa Cruz (Texas, USA). AntiBax, anti-Bcl-2, anti-caspase 3, anti-caspase 9, anti-cyclin D1, anti-cyclin E1, anti-phospho-ATM (pATM) (Ser1981), anti-ATM and anti- $\gamma$ H2A.X (Ser139) were purchased from Abcam (Cambridge, UK). Anti- $\beta$-actin, anti-GAPDH and HRP-conjugated goat anti-mouse/rabbit secondary antibodies were purchased from ComWin Biotech (Beijing, China).

\section{Real-time PCR}

Total RNA was isolated from NK-92 cells treated with REV or DMSO using TRIzol reagent (life technologies, Carlsbad, USA). RNA concentrations were detected on Nanodrop (Thermo Fisher, Waltham, USA). cDNA was synthesized using ReverTra Ace qPCR RT Kit (Toyobo, Osaka, Japan) according to manufacturer's introduction. Real-time PCR was performed on a CFX96 Touch $^{\mathrm{TM}}$ Real-Time PCR Detection System using SYBR Green Mix (Toyobo, Osaka, Japan). $\beta$-actin was used as a loading control. The mRNA expression levels were calculated according to the following formula:

The relative expression $=2^{-\Delta \Delta \mathrm{CT}}$, where $\Delta \mathrm{CT}=$ cycle threshold (CT) (detected gene) $-\mathrm{CT}(\beta$-actin) and $\Delta \Delta \mathrm{CT}=$ $\Delta \mathrm{CT}(\mathrm{REV})-\Delta \mathrm{CT}$ (control).

The primers were as follows: $\beta$-actin-forward:
5 '-ACTGGAACGGTGAAGGTGACAG-3', $\beta$-actinreverse: 5'-GGTGGCTTTTAGGATGGCAAG-3'; PLK1-forward: 5'-GCAGCGTGCAGATCAACTTC-3', PLK1-reverse: 5'-AGGAGACTCAGGCGGTATGT-3'.

\section{Hematoxylin and eosin (HE) and immunobistochemical (IHC) staining}

Paraffin wax embedded sections $(4 \mu \mathrm{m})$ were stained with HE. IHC staining was performed to detect pATM, ATM, pChk2, pp53 and ZEBRA protein using Envision method. The results were reviewed by two pathologists of Huashan Hospital. Proteins were scored based on intensity and distribution according to Dr. Li’s study (21).

\section{In situ bybridization (ISH)}

We detected the presence of EBV in NKTCL tissues by measuring EBV encoded small RNA (EBER) using ISH method. Slices were incubated with fluorescein-labeled EBER probe (Dako, Santa Clara, USA) and stained on an automated IHC and ISH systems Bond-III (Leica, Wetzlar, Germany) using Bond Polymer RedRefine Detection Kit (Leica, Wetzlar, Germany). Images were captured by Nikon DS-U3 imaging system (Nikon, Tokyo, Japan).

\section{Statistical analysis}

Data were presented as mean \pm standard error of the mean (SEM) from at least three independent experiments. $\mathrm{P}<0.05$ was considered as statistically significant.

Gene quantification of RNA-Seq data was performed with RSEM and FPKM method. Differentially expressed genes (DEGs) between two groups were screened using NOISeq method according to the following default criteria: Fold change $\geq 2$ and diverge probability $\geq 0.8$. Then we performed Gene Ontology (GO) enrichment analysis and KEGG Pathway Enrichment in the Database for Annotation, Visualization and Integrated Discovery (DAVID) (https:// david.ncifcrf.gov/). $\mathrm{P}<0.05$ was defined as significantly enriched GO terms and KEGG pathway.

The scores of pATM and ATM expression in NKTCL and normal lymph node tissues were analyzed according to rank sum test of non-parametric statistics using SPSS 19.0 software. $\mathrm{P}<0.05$ was considered statistically significant. The results were performed with GraphPad software (GraphPad software, San Diego, CA, USA). 


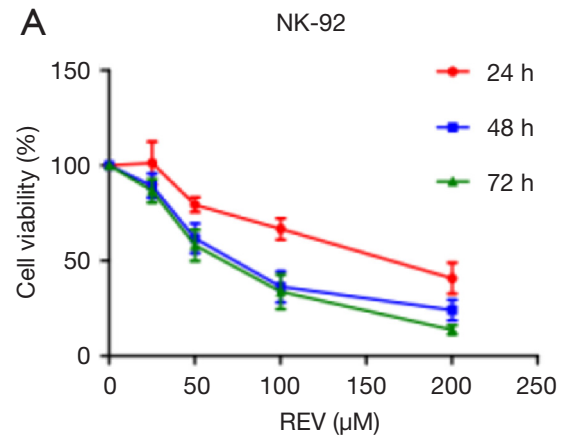

B

\begin{tabular}{cccc}
\hline Cell//C50 $(\mu \mathrm{M})$ & $24 \mathrm{~h}$ & $48 \mathrm{~h}$ & $72 \mathrm{~h}$ \\
\hline NK-92 & 154.00 & 75.44 & 65.21 \\
\hline
\end{tabular}

Figure 1 REV inhibited cell viability of NK-92 cells. (A) NK-92 cells were treated with REV in the concentrations of $0 \mu M$ (control), 25, 50, 100, or $200 \mu M$ for 24, 48 or $72 \mathrm{~h}$. The cell proliferation inhibition effect of REV was measured using CCK-8 assay (n=3). (B) IC50 of REV on NK-92 cells. REV, resveratrol; NKTCL, extranodal natural killer/T cell lymphoma.

\section{Results}

\section{REV inbibited proliferation of NK-92 cells}

We investigated the effects of REV on cell viability of NK-92 cells. The cells were treated with REV in different concentrations for different times. Cell viability was measured by CCK- 8 assay. The results showed that REV significantly inhibited the proliferation of NK-92 cells in a dose- and time-dependent manner (Figure 1A). IC50 of different treatment times was calculated (Figure 1B).

\section{REV arrested cell cycle of NK-92 cells at G1 phase}

Using flow cytometry to detect the cell cycle distribution change in REV-treated NK-92 cells by PI staining, we found that the percentage of G1 phase increased significantly after treatment with $100 \mu \mathrm{M} \mathrm{REV}$ for $24 \mathrm{~h}$ (Figure 2A). Further, we measured the expression level of cyclin D1 and cyclin E1, which are important molecules for cells transferring from $\mathrm{G} 1$ to $\mathrm{S}$ phase. The results demonstrated that REV decreased the level of cyclin D1 and cyclin E1 in NK-92 cells (Figure 2B). These data suggested REV arrested NK-92 cell cycle at G1 phase.

\section{REV induced mitochondrial apoptosis in NK-92 cells}

The apoptosis of cells was measured by using Alexa Fluor ${ }^{\circledR} 488$ Annexin V-/Dead Cell Apoptosis kit according to manufacturer's introduction. The data demonstrated that REV increased apoptotic percentage of cells in a dosedependent manner (Figure 3A). To explore the mechanism of REV inducing NK-92 cell apoptosis, the expression of apoptosis-related proteins was analyzed. Western blot analysis displayed that REV inhibited the expression of Bcl-2 while promoted the expression of Bax and Bad. It also promoted the cleavage of caspase 9 and caspase 3 (Figure 3B). These results suggested that REV induced NK-92 cell apoptosis through mitochondria-mediated apoptotic pathway.

\section{REV inbibited the expression of pAkt and pStat 3}

We next observed the expression of pAkt and pStat3 in REV treated cells. The results showed that the phosphorylation level of Akt was significantly decreased as early as $0.5 \mathrm{~h}$ after $\mathrm{REV}$ treatment. And pStat 3 was significantly diminished after $3 \mathrm{~h}$ of REV treatment (Figure $4 A$ ). These results suggested REV inhibited Akt and Stat3 pathways.

\section{REV activated DDR pathway in NK-92 cells}

To observe the effect of REV on DDR in NK-92 cells, we detected the expression of DDR marker proteins pATM, pChk2 and $\gamma$ H2A.X. As shown in Figure 4B, REV could significantly increase the expression levels of pATM, pChk2 and $\gamma \mathrm{H} 2 \mathrm{~A}$.X. These results suggested that REV activated DDR pathway in NK-92 cells.

\section{EBV infection was highly related with NKTCL}

HE staining of tissue slices showed a diffused distribution of lymphocyte in NKTCL tissues, and usually associated with necrosis (Figure $5 A$ ). ISH results showed that all cases expressed EBER (Figure 5B), indicating 100\% EBV infection in these 12 NKTCL cases. But the expression of EBV lytic gene, ZEBRA, was negative in all of the 12 cases, suggesting latency infection of EBV (Figure 5C). 


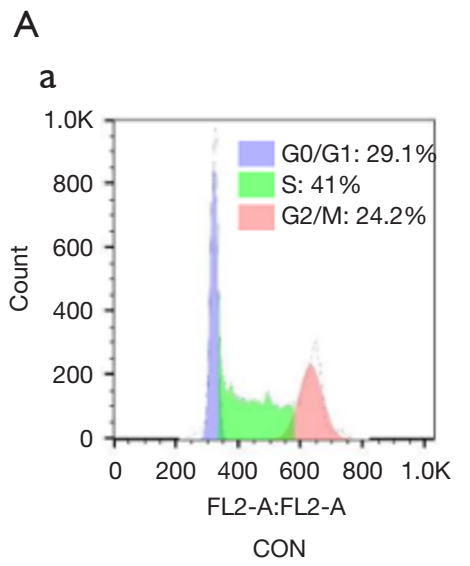

b
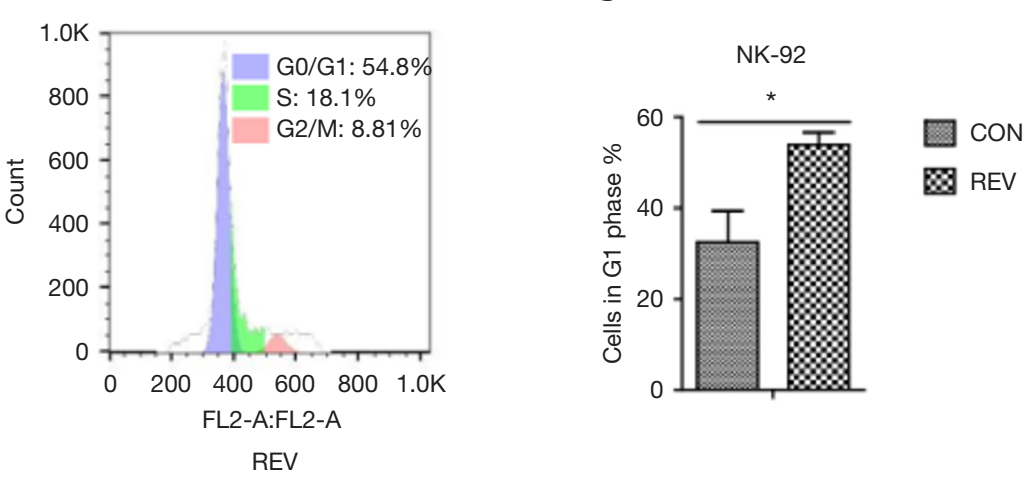

B

NK-92 (REV $100 \mu \mathrm{M})$

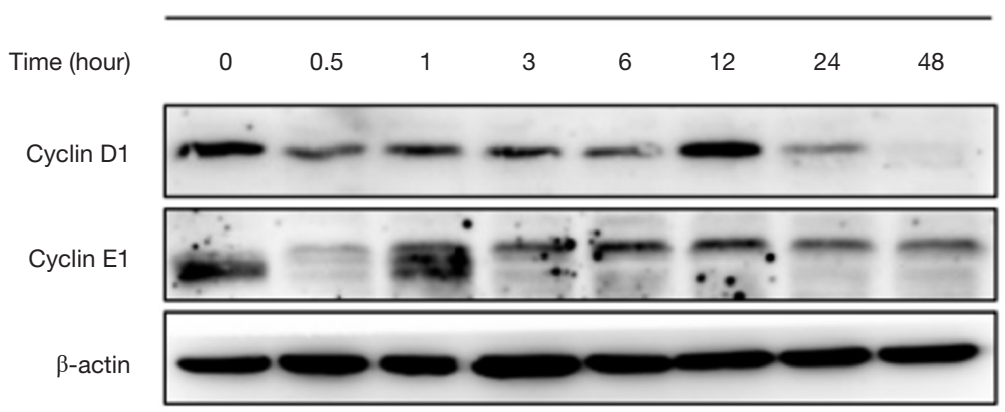

Figure 2 REV arrested NK-92 cell cycle at G1 phase. (A) DNA content of cells treated with REV for 24 h was measured by flow cytometry after PI staining: a. changes of cell proportion of different phases are shown; b. after treatment with REV, the proportion of NK-92 cells in G1 phrase raised from $29.1 \%$ to $54.8 \%$. *, P<0.05 vs. control. (B) Western blot analysis of the expression levels of cyclin D1 and cyclin E1 in cells treated with REV for different time. $\beta$-actin was used as a loading control. REV, resveratrol.

\section{ATM but not Chk2 or p53 is activated in buman NKTCL tumor tissues}

The IHC results demonstrated that pATM was distinguishingly expressed in different NKTCL samples. Eleven of the 12 cases were positive. While in all normal lymph nodes pATM was negative, as shown in Figure 6A. The expression level of pATM was significantly different in two groups $(\mathrm{P}=0.028)$ (Figure $6 B)$. ATM was negative or weakly positive in NKTCL while positive in normal lymph nodes $(\mathrm{P}=0.081)$ (Figure 6A,B). These results suggested that ATM is activated in NKTCL patients while inactive in normal lymph nodes.

However, pChk2 expression was weak in one case and negative in the rest 11 cases, and pp53 expression was very weak in four cases and negative in the rest eight cases, suggesting weak activation of DDR in NKTCL patients even ATM is highly phosphorylated (Figure 6C).

\section{PLK1 is overexpressed in NKTCL tumor tissues and NKTCL cell lines}

We searched the DEGs in the array transcription profiling database E-TABM-702 in Array Express containing 26 normal tissue samples, 7 NKTCL tissue samples and 2 NKTCL cell lines. The PLK1 expression in NKTCL tissues and cell lines was greatly elevated $(\mathrm{P}<0.05)$ (Figure $6 D$ ).

\section{DEGs of REV treated NK-92 cells}

We used RNA-seq technology to screen DEGs between REV and control groups in NK-92 cells, and then performed functional annotation analysis. Most of the REV 
A

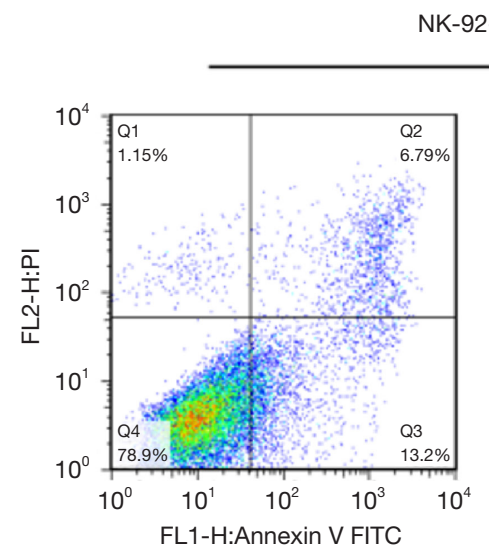

$0 \mu \mathrm{M}$

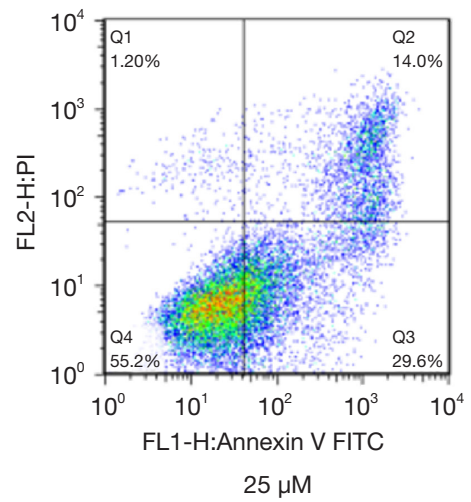

$25 \mu \mathrm{M}$

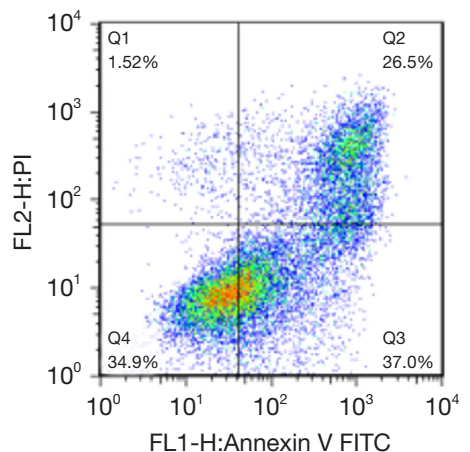

$50 \mu \mathrm{M}$

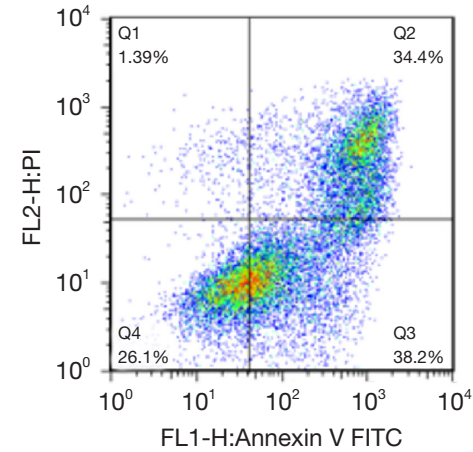

$100 \mu \mathrm{M}$

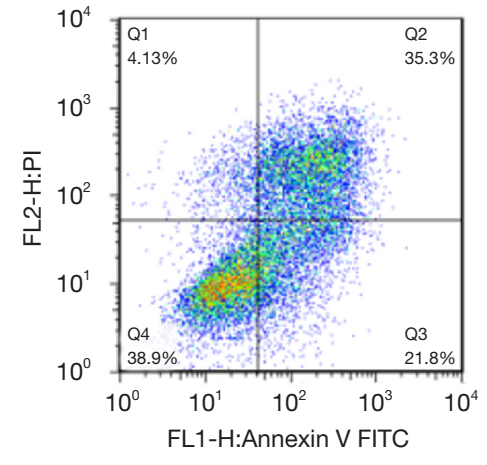

$200 \mu \mathrm{M}$

B

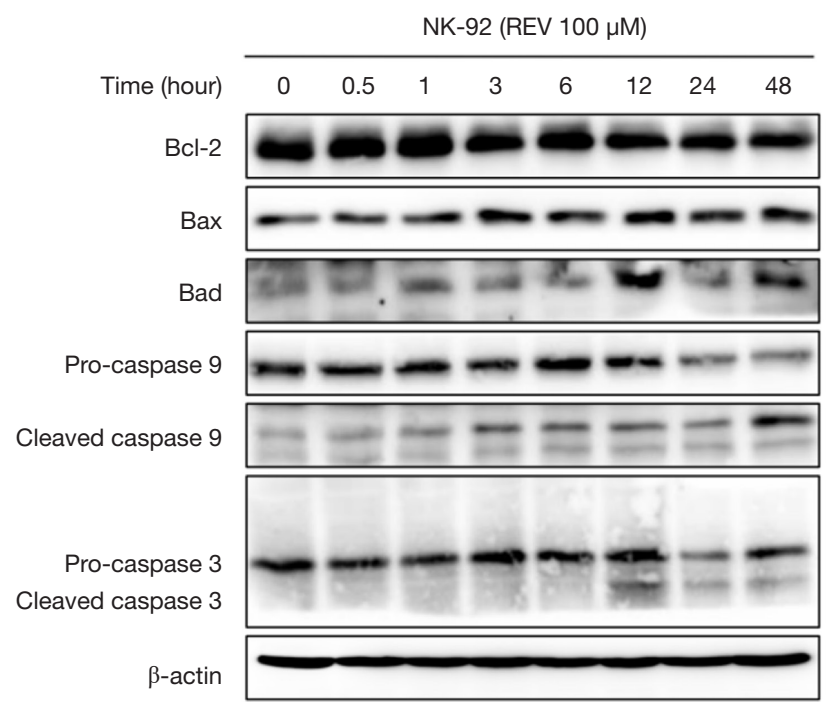

Figure 3 REV induced apoptosis of NK-92 cells through the mitochondria-mediated apoptotic pathway. (A) Cells were treated with different concentrations of REV for $48 \mathrm{~h}$. The apoptosis was measured by flow cytometry using Annexin V-FITC apoptosis detection kit. (B) The protein expression levels of Bcl-2, Bax, Bad, caspase 9, cleaved caspase 9, caspase 3, and cleaved caspase 3 in NK-92 cells treated with REV for different time were measured by Western blot. $\beta$-actin was used as a loading control. REV, resveratrol. 
A

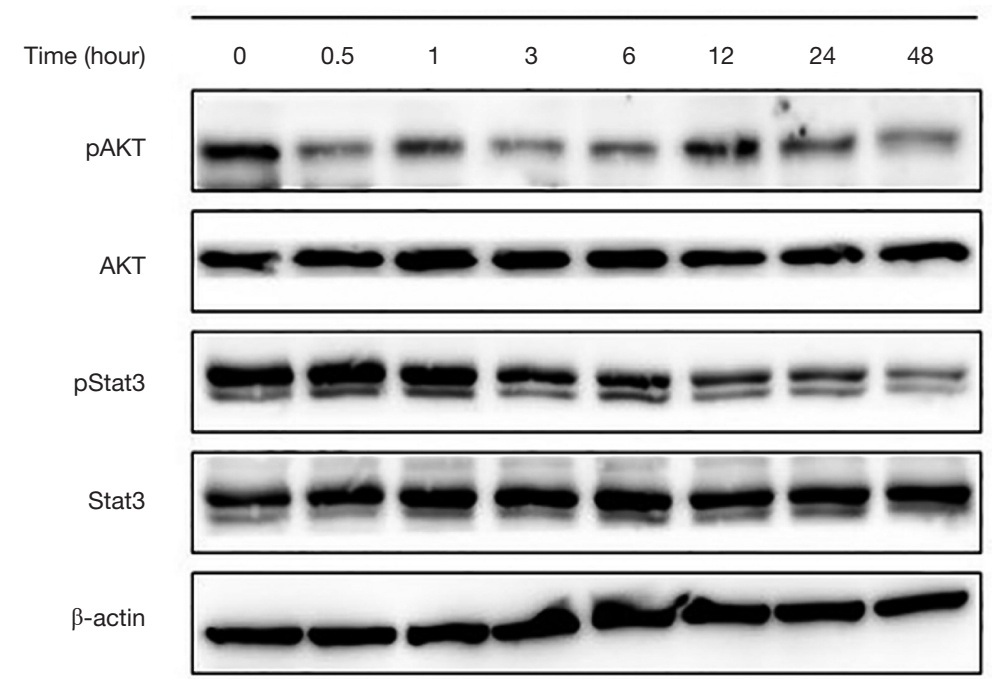

B

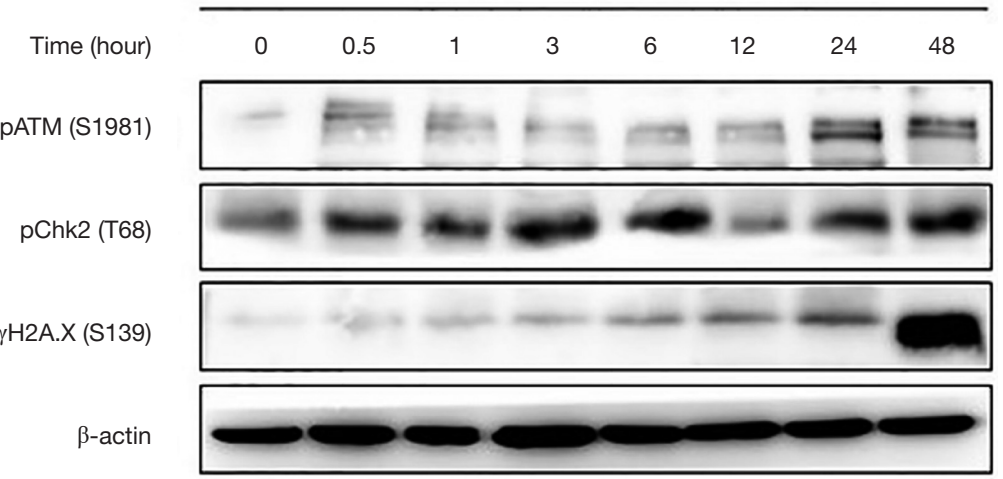

Figure 4 The expression of proteins involved in DDR and pAkt and pStat3 pathways was analyzed by Western blot. (A) REV inhibited the expression of pAkt and pStat3. (B) REV promoted the expression of DDR related proteins pATM, pChk2 and $\gamma \mathrm{H} 2 \mathrm{~A}$.X. $\beta$-actin was used as a loading control. REV, resveratrol.

regulated genes are core controllers of cell cycle, apoptosis, PI3K-Akt, MAPK and p53 signal pathways (Figure 7A). Interestingly, these pathways are all related to DDR. It suggests that DDR may play an important role in REV inhibiting NKTCL proliferation. Among these DEGs, PLK1, a highly conserved serine-threonine kinase, which combines with and dephosphorylates Chk2 and p53, was down-regulated significantly in NK-92 cells after treatment with REV (Figure 7B).

\section{REV activated DDR patbway through down-regulating PLK1}

Using real-time PCR, we could see REV inhibited the
mRNA expression level of PLK1 in NK-92 cells (Figure 8A). Using Western blot analysis, we found that REV inhibited the expression of both PLK1 and pPLK1 (Figure 8B). Further, REV increased the phosphorylation level of p 53 and the expression of p21 (Figure 8B), resulting in cell apoptosis.

\section{Discussion}

NKTCL progresses aggressively. Distant dissemination usually occurs early in the clinical course. Because of the low incidence, randomized control trials on treatment of NKTCL are limited. Most treatment protocols are consensus based and are derived from retrospective and 
A a

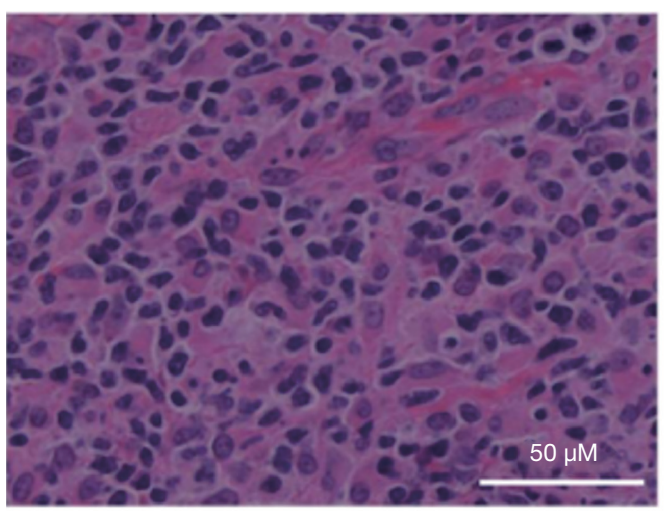

NKTCL

B

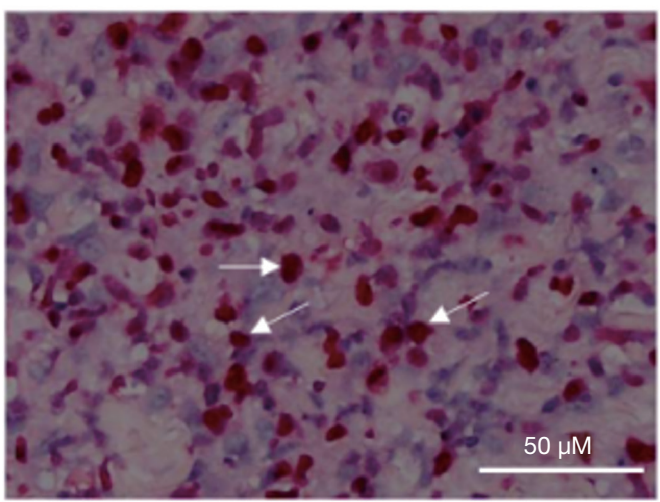

EBER b

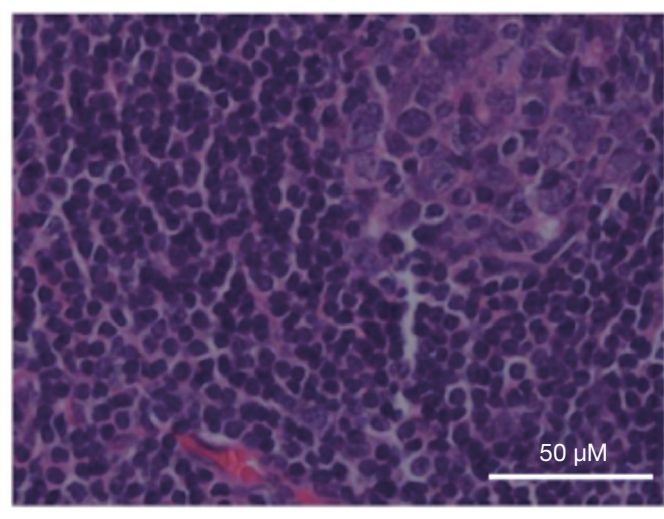

Normal lymph node

C

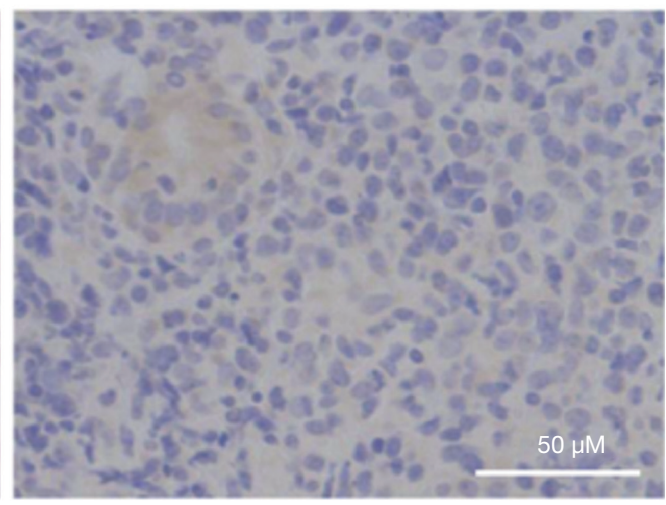

ZEBRA

Figure 5 The HE staining and ISH in human NKTCL tumor tissues. (A) HE staining of NKTCL tissues and normal lymph nodes: a. HE staining of NKTCL tissues; b. HE staining of normal lymph nodes. IHC and immunohistochemistry assay of EBV: (B) IHC staining of EBER in NKTCL tissues; (C) immunohistochemistry test of ZEBRA in NKTCL tissues. NKTCL, extranodal natural killer/T cell lymphoma; ISH, in situ hybridization; IHC, immunohistochemistry; EBER, Epstein-Barr virus encoded small RNA;

small prospective studies (22). The prognosis of NKTCL has been gradually improved with modern treatment protocols combining chemotherapy and radiotherapy. But the outcome is still far from satisfactory due to the high rate of disease progression and relapse, as well as intolerable hematologic toxicity. Therefore, exploration for effective treatment is needed for NKTCL. The traditional Chinese drug, REV, may be competent for this job.

REV, a polyphenolic compound rich in grapes, blueberries, and peanuts, has multiple pharmacological activities. Recently, the antitumor activity of REV is gaining increasingly interest. REV shows anticarcinogenic effects in many aspects, including inhibiting tumor cell growth, inducing inflammation and apoptosis, inhibiting angiogenesis and tumor invasion and/or metastasis, reversing drug resistance and sensitizing tumor cells to radiotherapies and chemotherapies $(23,24)$. Moreover, REV can induce cell cycle arrest and apoptosis of four malignant NK cells by inhibiting JAK2-Stat3 pathway (25). By increasing reactive oxygen species (ROS) level, REV caused DNA damage and activated DDR in colon cancer cells (26). In glioblastoma, prostate cancer and breast cancer cells, REV induced apoptosis through activating p53 and its downstream target genes (27-29).

In our study, REV inhibited cell proliferation, and arrested cell cycle at G1 phase in NK-92 cells. Moreover, $\mathrm{REV}$ induced cell apoptosis in dose-dependent manner as measured by flow cytometry. Western Blot analysis 
A

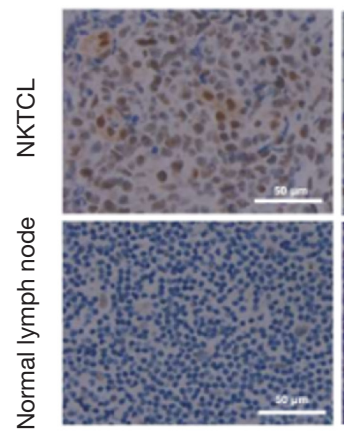

PATM

C
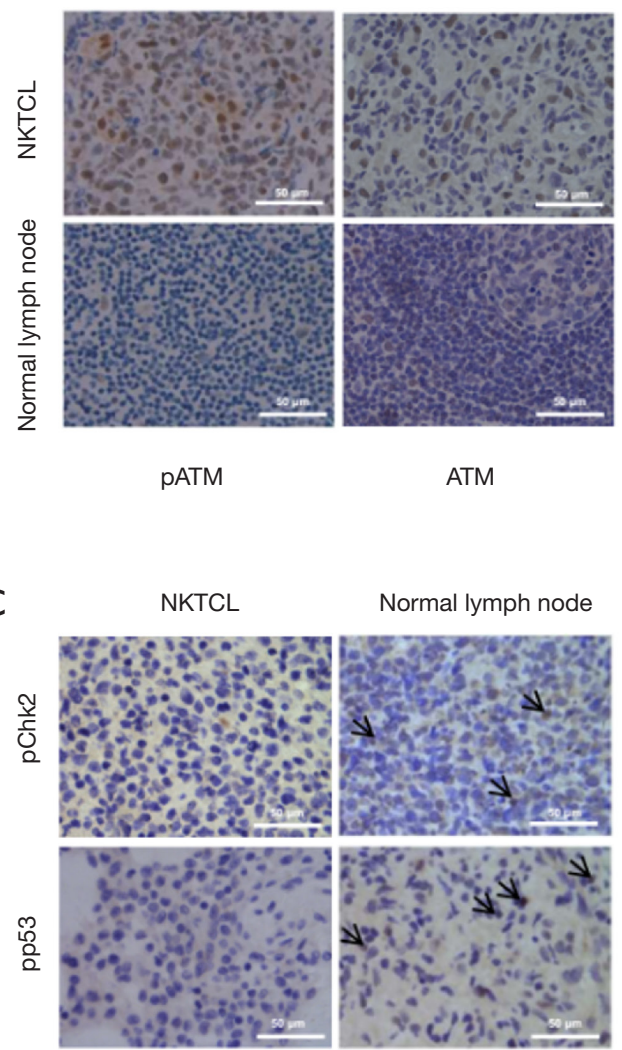

ATM
B
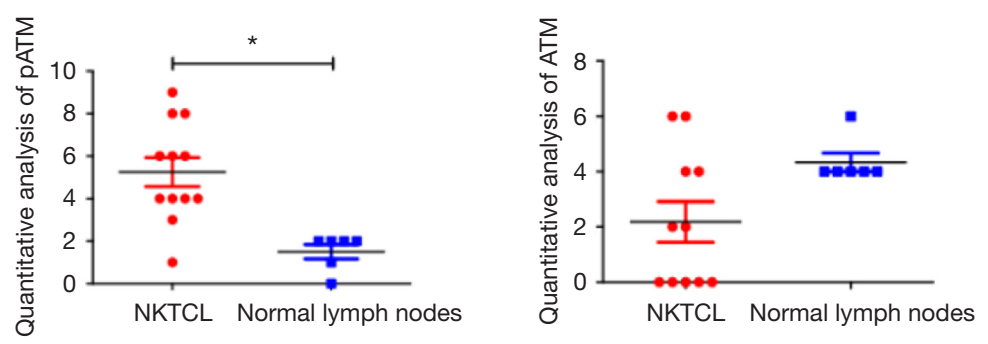

$\mathrm{D}$

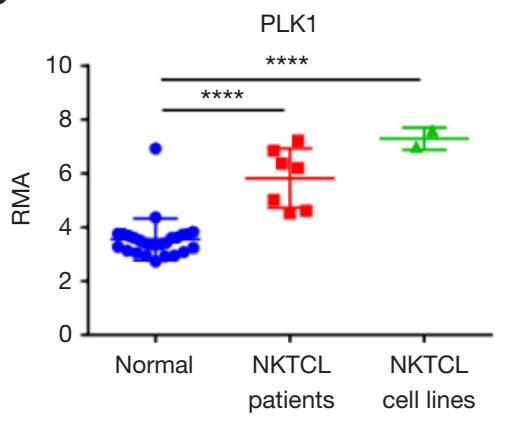

Figure 6 pATM was activated while pChk2 and pp53 were weakly activated and PLK1 is overexpressed in human NKTCL tumor tissues. $(\mathrm{A}, \mathrm{B})$ Immunohistochemistry tests of PATM and ATM in NKTCL tissues and normal lymph nodes: (A) immunohistochemistry tests of pATM and ATM in NKTCL tissues and normal lymph nodes; (B) analysis of the expression of PATM and ATM in NKTCL tissues and normal lymph nodes. *, $\mathrm{P}<0.05$ compared to normal lymph nodes. (C) Immunohistochemistry tests of pChk2 and pp53 in NKTCL tissues and normal lymph nodes. (D) Analysis of a tissue chip result from an online database Array Express, the ID of which is E-TABM-702, containing 26 normal tissue samples, 7 NKTCL tissue samples and 2 NKTCL cell lines. The expression level of PLK1 in NKTCL patients' tissues was higher than normal tissues $\left.{ }^{* * * *}, \mathrm{P}<0.0001\right)$, and was even higher in NKTCL cell lines $\left.{ }^{* * * *}, \mathrm{P}<0.0001\right)$.

displayed that REV inhibited the expression of Bcl-2 while promoted the expression of Bax and Bad. It also promoted the cleavage of caspase 9 and caspase 3. These results suggested that REV induced NK-92 cell apoptosis through mitochondria-mediated apoptotic pathway. Further, we detected the activation of DDR and the inhibition of AKT and JAK-Stat pathways by REV in NK-92 cell line, which indicated that these pathways were targeted by REV.

DDR is essential for maintaining the genomic integrity of cells, which results in DNA repair, chromatin remodeling, cell cycle arrest or cell apoptosis under different conditions (13). When the DNA damage happens, H2A. $\mathrm{X}$, served as a first step sensor activates, following with a key mediator, ATM activated through autophosphorylation of serine 1,981 , which subsequently activates a range of downstream targets involved in cell cycle control, apoptotic response and DNA repair, such as Chk2, p53, cyclins, etc. (8). Unless repaired in an error-free process, DNA damage can result in mutations and altered cellular behavior (30). The downregulation of DNA damage surveillance and up-regulation of genomic instability lead to tumor development (31). Thus, DDR disruption is one of the hallmarks of cancer for inactivation of DDR pathway, typically leads to increased genomic instability (30).

Disruption of the DDR is observed in many cancers to escape from apoptosis and stabilize DNA lesions, such as prostate cancer and breast cancer (26,27). Even in most cases, the specific underlying defects are poorly 
A

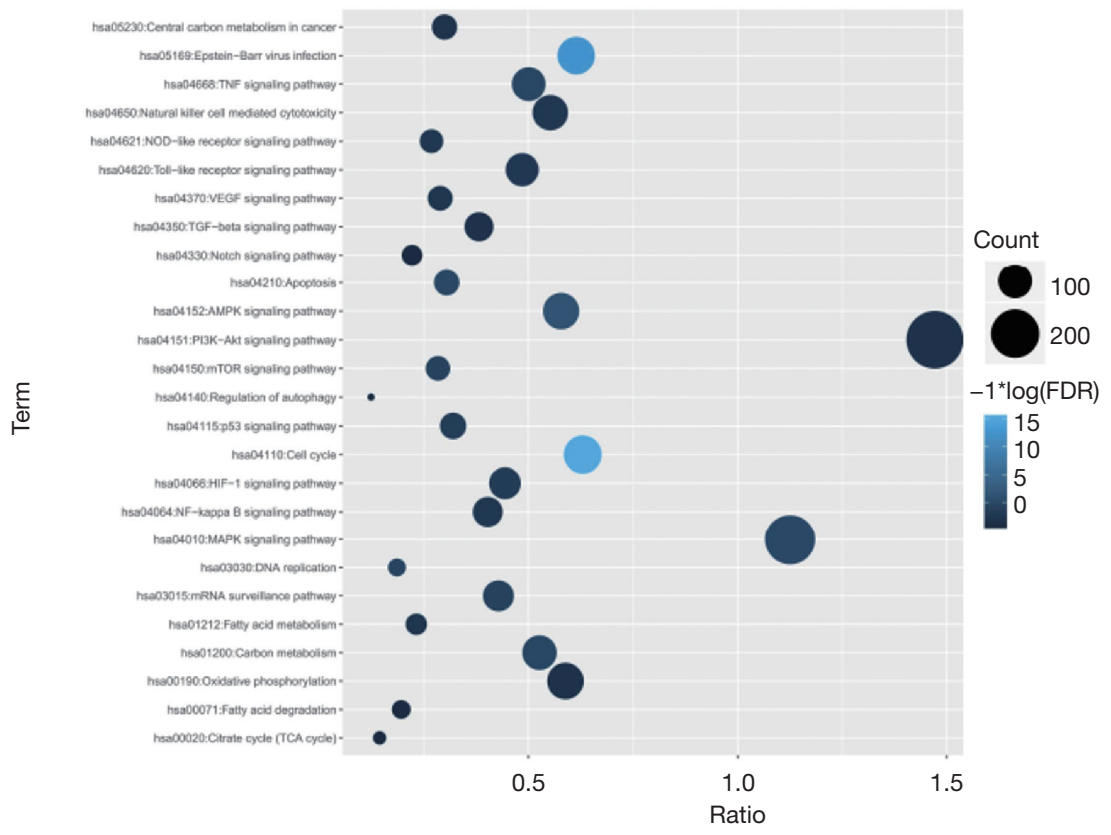

B

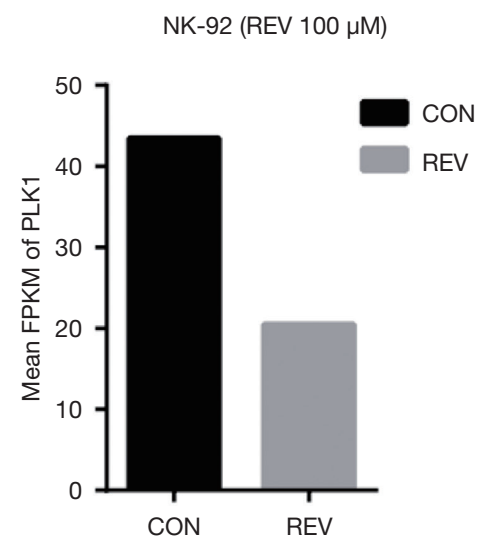

Figure 7 REV regulated key genes and pathways in NK-92 cell lines based on RNA-seq results. (A) After treatment with REV for 6 h, the differentially expressed genes were screened using RNA-seq method in NK-92 cells. KEGG pathway enrichment analyses of the DEGs conducted using DAVID website showed that most of the DEGs are concerning cell cycle, apoptosis and p53, which are all related to DDR. (B) The mRNA expression level of PLK1 in NK-92 cells treated with $100 \mu \mathrm{M} \mathrm{REV} \mathrm{for} 6 \mathrm{~h}$ was measured using RNA-seq technology. The mean of three determinations of FPKM was presented. REV, resveratrol; DEGs, differentially expressed genes; DDR, DNA damage response; PLK1, polo-like kinase 1.

characterized. In our study, we measured DDR in 12 NKTCL patients and 6 normal lymph nodes. The higher expression level of pATM in NKTCL samples than in normal lymph nodes indicated the beginning of DDR activation. On the other hand, the downstream molecules of ATM, both Chk2 and p53 were scarcely activated, which suggests the disruption of DDR in NKTCL patients even ATM is highly phosphorylated.

These results indicate that there is a blockage from activation of ATM to activation of Chk2. However, our data showed REV activated DDR pathway in NK-92 cells through enhancing the phosphorylation level of ATM, Chk2 and H2A.X, indicating that REV could get through the barrier from ATM activation to Chk2 activation. Although the expression of $\mathrm{p} 53$ protein is constant in NKTCL cells treated by REV, which was exactly consistent with Quoc Trung L's work (25), the level of pp53 was increased after REV treatment, which could activate p21, and result in cell proliferation inhibition, cell cycle arrest and apoptosis.

To further study the targets of REV in reactivating
DDR, we turned to the RNA-seq method to screen DEGs between REV and control groups in NK-92 cells. After treatment with REV for $6 \mathrm{~h}$, NK-92 cells showed DEGs concerning cell cycle, apoptosis and p53, which are all related to DDR.

Among these DEGs, PLK1 was downregulated significantly in NK-92 cells after treatment with REV. PLKs are a family of serine/threonine protein kinases including five members: PLK1, PLK2, PLK3, PLK4, and PLK5. Among them, PLK1 is essential for precisely regulating cell division and maintaining genome stability in mitosis, spindle assembly, and DDR (28). When the damaged DNA is well repaired and the cell is ready to go on its life span, PLK1 inhibits the key components of DDR, Chk2 and p53, to allow the recovery from cell cycle checkpoint (29). At this process, PLK1 physically binds to Chk2 to deactivate it through the phosphorylation of the FHA domain (32), and binds to p53 to inhibit its transactivation activity as well as its pro-apoptotic function (33).

PLK1 is abnormally overexpressed in tumors, which 
A

NK-92 (REV $100 \mu \mathrm{M})$

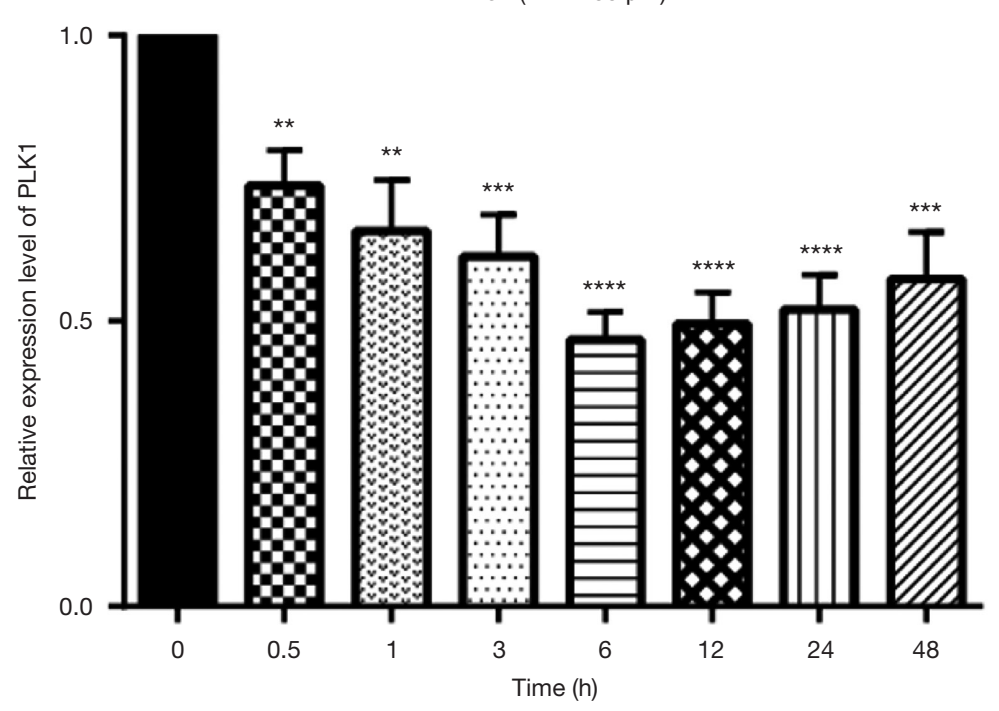

B

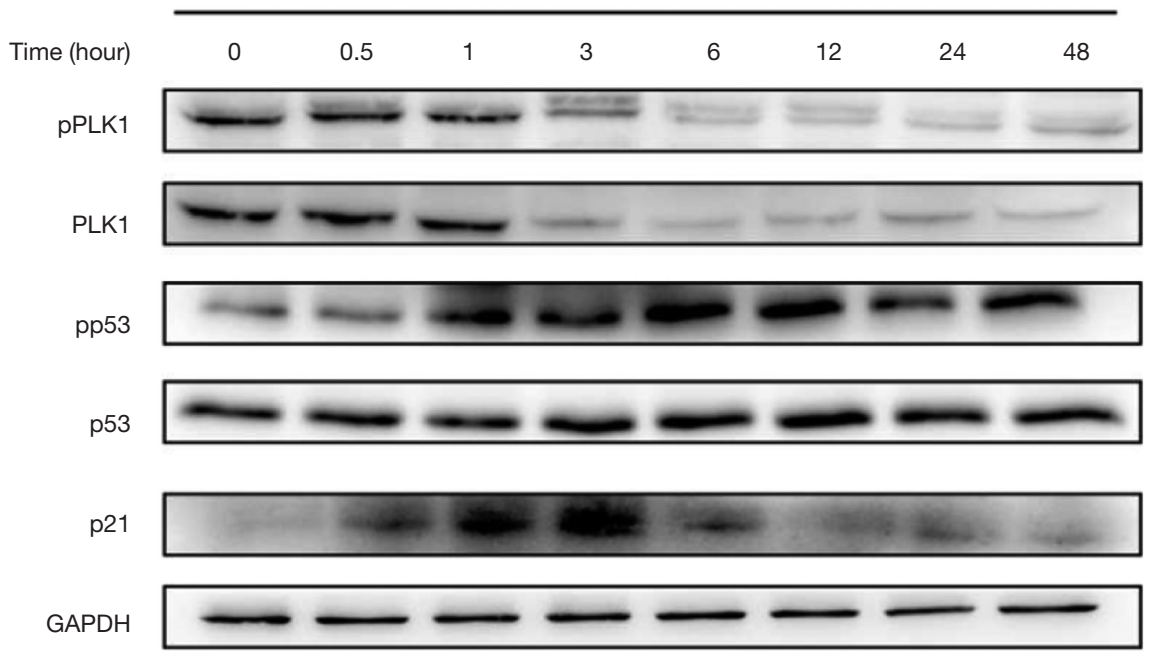

Figure 8 REV activated DDR pathway through down-regulating PLK1. (A) REV inhibits PLK1 expression. The mRNA expression levels of PLK1 in NK-92 cells treated with $100 \mu \mathrm{M}$ REV for different time were measured using real-time PCR. (B) The expression levels of PLK1, pPLK1, pp53 and p21 were measured using Western blot analysis after treatment with $100 \mu M$ REV for different hours. GAPDH was used as loading control. ${ }^{* *}, \mathrm{P}<0.01$; ${ }^{* * *}, \mathrm{P}<0.001$; ${ }^{* * *}, \mathrm{P}<0.0001$ compared to control group. REV, resveratrol; PLK1, polo-like kinase 1; DDR, DNA damage response.

might explain how tumor cells escape from death fate caused by the should-be activated DDR $(10,29)$. Blocking PLK1 can effectively inhibit the proliferation and induce apoptosis of tumor cells (34). Thus, it has been suggested that PLK1 could be an attractive target for cancer therapy. In our study, we analyzed an online database, ID E-TABM-702 in Array Express, and found that PLK1 expression in NKTCL tissues and cell lines were greatly elevated. After treatment with REV, both the expression of PLK1 and its phosphorylation level were inhibited, which resulted in the activation of Chk2 and p53.p53 then up-regulated cell cycle inhibitors such as the CDK inhibitor p21, leading to cell cycle arrest at G1 through down-regulation of cyclin D1 and E1. Also, p53 induced cell apoptosis though Bcl-2 family and finally activated caspase cascade. These results suggest that REV activates DDR pathway through down- 


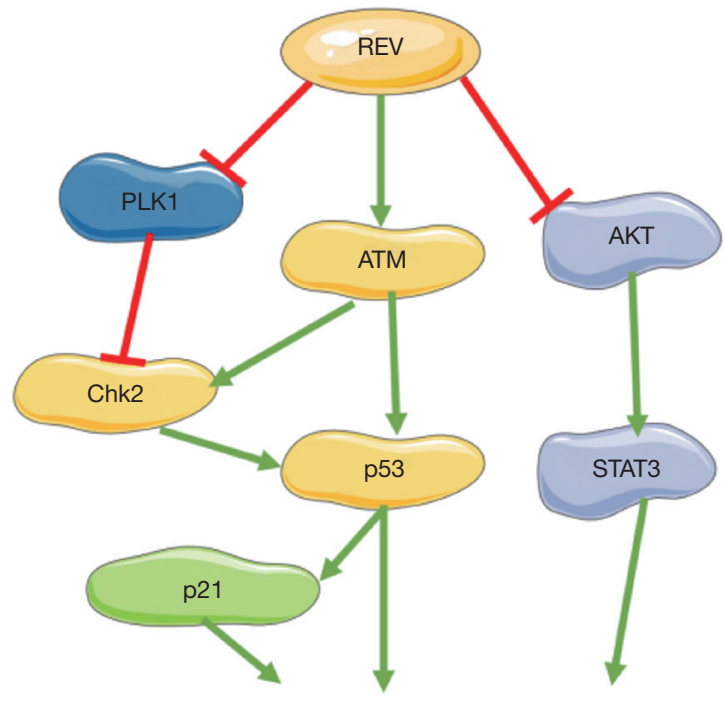

Inhibition of cell proliferation, induction of cell cycle arrest and apoptosis

Figure 9 A simplified scheme of REV inhibiting NKTCL cells. REV inhibits NKTCL cell proliferation and induces G1 phase arrest and apoptosis. REV induces DDR by activating ATM, which phosphorylates H2A.X, Chk2 and p53, ultimately activates p21. PLK1 inhibited by REV releases Chk2 activity. AKT and STAT3 inhibited by REV also contributes to anti-tumor effect. REV, resveratrol; DDR, DNA damage response; NKTCL, extranodal natural killer/T cell lymphoma; Chk2, checkpoint kinase 2; PLK1, polo-like kinase 1 .

regulation of PLK1, ultimately results in NKTCL cell apoptosis.

Taken together, in NKTCL patients, DDR is disrupted even ATM is highly phosphorylated. REV can activate DDR pathway through inhibition of PLK1, inhibit AKT and Stat3, ultimately show anti-tumor effect in NKTCL cells (Figure 9). Due to its low toxicity in animal models and in humans, REV can be a potential anti-tumor drug worthy of further study for treating NKTCL.

\section{Acknowledgments}

Funding: The study was supported by the National Natural Science Foundation of China (Grant No. 81470352 and 81770208).

\section{Footnote}

Data Sharing Statement: Available at http://dx.doi. org/10.21037/atm-19-4324

Conflicts of Interest: All authors have completed the ICMJE uniform disclosure form (available at http://dx.doi. org/10.21037/atm-19-4324). The authors have no conflicts of interest to declare.

Ethical Statement: The authors are accountable for all aspects of the work in ensuring that questions related to the accuracy or integrity of any part of the work are appropriately investigated and resolved. This study was approved by medical ethics committee of school of Basic Medical Sciences, Fudan University (No. 2017-C003).

Open Access Statement: This is an Open Access article distributed in accordance with the Creative Commons Attribution-NonCommercial-NoDerivs 4.0 International License (CC BY-NC-ND 4.0), which permits the noncommercial replication and distribution of the article with the strict proviso that no changes or edits are made and the original work is properly cited (including links to both the formal publication through the relevant DOI and the license). See: https://creativecommons.org/licenses/by-nc-nd/4.0/.

\section{References}

1. WHO Classification of Tumours of Haematopoietic and Lymphoid Tissues. 4th edition. Lyon, France: IARC, 2017.

2. Yamaguchi M. Current and future management of NK/ T-cell lymphoma based on clinical trials. Int J Hematol 2012;96:562-71.

3. Yamaguchi M, Oguchi M, Suzuki R. Extranodal NK/ T-cell lymphoma: Updates in biology and management strategies. Best Pract Res Clin Haematol 2018;31:315-21.

4. Lim SH, Hong JY, Lim ST, et al. Beyond first-line non-anthracycline-based chemotherapy for extranodal NK/T-cell lymphoma: clinical outcome and current perspectives on salvage therapy for patients after first relapse and progression of disease. Ann Oncol 2017;28:2199-205.

5. Liu A, Takakuwa T, Luo WJ, et al. Alterations in ATR in nasal NK/T-cell lymphoma and chronic active EpsteinBarr virus infection. Cancer Sci 2006;97:605-10.

6. Maréchal A, Zou L. DNA damage sensing by the ATM and ATR kinases. Cold Spring Harb Perspect Biol 2013;5:a012716.

7. Davalli P, Marverti G, Lauriola A, et al. Targeting 
Oxidatively Induced DNA Damage Response in Cancer: Opportunities for Novel Cancer Therapies. Oxid Med Cell Longev 2018;2018:2389523.

8. Matt S, Hofmann TG. The DNA damage-induced cell death response: a roadmap to kill cancer cells. Cell Mol Life Sci 2016;73:2829-50.

9. Jaiswal H, Benada J, Müllers E, et al. ATM/Wip1 activities at chromatin control Plk1 re-activation to determine G2 checkpoint duration. EMBO J 2017;36:2161-76.

10. Eckerdt F, Yuan J, Strebhardt K. Polo-like kinases and oncogenesis. Oncogene 2005;24:267-76.

11. Chen Z, Chai Y, Zhao T, et al. Effect of PLK1 inhibition on cisplatin-resistant gastric cancer cells. J Cell Physiol 2019;234:5904-14.

12. Wang Y, Wu L, Yao Y, et al. Polo-like kinase 1 inhibitor BI 6727 induces DNA damage and exerts strong antitumor activity in small cell lung cancer. Cancer Lett 2018;436:1-9.

13. Hosoya N, Miyagawa K. Targeting DNA damage response in cancer therapy. Cancer Sci 2014;105:370-88.

14. Elshaer M, Chen Y, Wang XJ, et al. Resveratrol: An overview of its anti-cancer mechanisms. Life Sci 2018;207:340-9.

15. Yousef M, Vlachogiannis IA, Tsiani E. Effects of Resveratrol against Lung Cancer: In Vitro and In Vivo Studies. Nutrients 2017;9:1231-45.

16. Ko JH, Sethi G, Um JY, et al. The Role of Resveratrol in Cancer Therapy. Int J Mol Sci 2017;18:2589.

17. Gatz SA, Keimling M, Baumann C, et al. Resveratrol modulates DNA double-strand break repair pathways in an ATM/ATR-p53- and -Nbs1-dependent manner. Carcinogenesis 2008;29:519-27.

18. Carter LG, D'Orazio JA, Pearson KJ. Resveratrol and cancer: focus on in vivo evidence. Endocr Relat Cancer 2014;21:R209-25.

19. Boocock DJ, Faust GE, Patel KR, et al. Phase I dose escalation pharmacokinetic study in healthy volunteers of resveratrol, a potential cancer chemopreventive agent. Cancer Epidemiol Biomarkers Prev 2007;16:1246-52.

20. Fulda S, Debatin KM. Sensitization for anticancer drug-induced apoptosis by the chemopreventive agent resveratrol. Oncogene 2004;23:6702-11.

21. Li J, Yin M, Song W, et al. B Subunit of Human Chorionic Gonadotropin Promotes Tumor Invasion and Predicts Poor Prognosis of Early-Stage Colorectal Cancer. Cell Physiol Biochem 2018;45:237-49.

22. Gill H, Liang RH, Tse E. Extranodal natural-killer/t-cell lymphoma, nasal type. Adv Hematol 2010;2010:627401.

23. Mondal A, Bennett LL. Resveratrol enhances the efficacy of sorafenib mediated apoptosis in human breast cancer MCF7 cells through ROS, cell cycle inhibition, caspase 3 and PARP cleavage. Biomed Pharmacother 2016;84:1906-14.

24. Wang S, Meng Q, Xie Q, et al. Effect and mechanism of resveratrol on drug resistance in human bladder cancer cells. Mol Med Rep 2017;15:1179-87.

25. Quoc Trung L, Espinoza JL, Takami A, et al. Resveratrol induces cell cycle arrest and apoptosis in malignant NK cells via JAK2/STAT3 pathway inhibition. PLoS One 2013;8:e55183.

26. Pearl LH, Schierz AC, Ward SE, et al. Therapeutic opportunities within the DNA damage response. Nat Rev Cancer 2015;15:166-80.

27. Jeggo PA, Pearl LH, Carr AM. DNA repair, genome stability and cancer: a historical perspective. Nat Rev Cancer 2016;16:35-42.

28. Karanika S, Karantanos T, Li L, et al. DNA damage response and prostate cancer: defects, regulation and therapeutic implications. Oncogene 2015;34:2815-22.

29. Ali R, Rakha EA, Madhusudan S, et al. DNA damage repair in breast cancer and its therapeutic implications. Pathology 2017;49:156-65.

30. Liu Z, Sun Q, Wang X. PLK1, A Potential Target for Cancer Therapy. Transl Oncol 2017;10:22-32.

31. van Vugt MA, Medema RH. Checkpoint adaptation and recovery: back with Polo after the break. Cell Cycle 2004;3:1383-6.

32. Zannini L, Delia D, Buscemi G. CHK2 kinase in the DNA damage response and beyond. J Mol Cell Biol 2014;6:442-57.

33. Ando K, Ozaki T, Yamamoto H, et al. Polo-like kinase 1 (Plk1) inhibits p53 function by physical interaction and phosphorylation. J Biol Chem 2004;279:25549-61.

34. de Oliveira JC, Brassesco MS, Pezuk JA, et al. In vitro PLK1 inhibition by BI 2536 decreases proliferation and induces cell-cycle arrest in melanoma cells. J Drugs Dermatol 2012;11:587-92.

Cite this article as: Sui X, Zhang C, Jiang Y, Zhou J, Xu C, Tang F, Chen B, Xu H, Wang S, Wang X. Resveratrol activates DNA damage response through inhibition of polo-like kinase 1 (PLK1) in natural killer/T cell lymphoma. Ann Transl Med 2020;8(11):688. doi: 10.21037/atm-19-4324 